\title{
Path integral approach to no-Coriolis approximation in heavy-ion collisions
}

\author{
K. Hagino, ${ }^{1}$ N. Takigawa, ${ }^{1}$, A.B. Balantekin ${ }^{2}$, and J.R. Bennett ${ }^{3}$ \\ ${ }^{1}$ Department of Physics, Tohoku University, 980-77 Sendai, Japan \\ 2 Physics Department, University of Wisconsin, \\ Madison, Wisconsin 53706, USA \\ 3 Department of Physics and Astronomy, \\ University of North Carolina at Chapel Hill, Chapel Hill, NC 27599-3255
}

\begin{abstract}
We use the two time influence functional method of the path integral approach in order to reduce the dimension of the coupled-channels equations for heavy-ion reactions based on the no-Coriolis approximation. Our method is superior to other methods in that it easily enables us to study the cases where the initial spin of the colliding particle is not zero. It can also be easily applied to the cases where the internal degrees of freedom are not necessarily collective coordinates. We also clarify the underlying assumptions in our approach.
\end{abstract}

PACS number(s): 25.70.Jj, 74.50.+r, 21.10.Re 
It is by now well established that heavy-ion fusion reactions at energies below the Coulomb barrier are not such simple processes that can be described in terms of penetration through a one dimensional potential barrier, but rather complicated reactions where internal degrees of freedom of colliding nuclei play an essential role[1]. Therefore they are typical examples of macroscopic quantum tunneling, which has been a very popular subject in the past decade in many subfields of physics and chemistry[2-4]. One of the major interests in macroscopic quantum tunneling is to assess the effects of the environment on the tunneling rate of a macroscopic degree of freedom. A standard way to tackle this problem in nuclear physics is to numerically solve the associated coupledchannels equations. However, the full coupled-channels calculations quickly become very intricate if many physical channels are included. This makes an intuitive understanding of the numerical results quite hard. For this reason, an approximation named the no-Coriolis approximation, which is sometimes called the rotating frame approximation, has been introduced[5-11]. It is a sudden tunneling approximation concerning the centrifugal energy and it greatly reduces the number of coupled channels to be solved. The no-Coriolis approximation was first introduced in the field of chemistry under the name centrifugal sudden approximation[13-15]. Recently, it has also been applied to the problem of electron-molecule scattering [16].

The no-Coriolis approximation has been derived by several different methods. The authors in ref.[5-8] used properties of Racah coefficients, and ref. [9, [0] used the Green's function method. Symmetry considerations using tidal spin have been used in ref. [11]. The aim of this paper is to present a new derivation of the no-Coriolis approximation using the path integral method[17]. This approach had already been used in ref. [18], but the angular momentum coupling was not treated explicitly. A salient point of our derivation is that it enables us to easily extend to the cases where the initial spin of the colliding 
particles is not zero, and where there is a spin-orbit force in the scattering process. It can also be easily applied to the cases where the internal degrees of freedom are not collective coordinates, but the coordinates of the constituent particles of the colliding system. It also clarifies the underlying assumption of the approximation.

We consider the collision between two nuclei in the presence of the coupling between the translational motion, i.e. the relative motion between the centers of mass of the colliding nuclei, $\boldsymbol{R}=(R, \Omega)$ and a nuclear intrinsic motion $\xi$. We assume the following Hamiltonian for this system

$$
H(\boldsymbol{R}, \xi)=-\frac{\hbar^{2}}{2 \mu} \nabla^{2}+U(R)+H_{0}(\xi)+V(\boldsymbol{R}, \xi)
$$

where $\mu$ is the reduced mass. $U(R), H_{0}(\xi)$ and $V(\boldsymbol{R}, \xi)$ are the bare potential energy for the translational motion, the internal Hamiltonian and the coupling Hamiltonian, respectively. In general the internal degree of freedom $\xi$ has a finite spin. We therefore expand the coupling Hamiltonian in multipoles

$$
V(\boldsymbol{R}, \xi)=\sum_{\lambda>0} f_{\lambda}(R) Y_{\lambda}(\Omega) \cdot T_{\lambda}(\xi) .
$$

Here $Y_{\lambda}(\Omega)$ are the spherical harmonics and $T_{\lambda}(\xi)$ are spherical tensors constructed from the internal coordinate. The dot indicates a scalar product. The sum is taken over all values of $\lambda$, except for $\lambda=0$ which is already included in $U(R)$.

For a fixed total angular momentum $J$ and its $z$ component $M$, the expansion basis of the coupled-channels equations are defined as

$$
<\Omega \xi\left|(n L I) J M>=\sum_{m_{L}, m_{I}}<L m_{L} \operatorname{Im}_{I}\right| J M>Y_{L m_{L}}(\Omega) \varphi_{n I m_{I}}(\xi),
$$

where $L$ and $I$ are the orbital and the internal angular momenta, respectively. $\varphi_{n I m_{I}}(\xi)$ are the wave functions of the internal motion which obey

$$
H_{0}(\xi) \varphi_{n I m_{I}}(\xi)=\epsilon_{n I} \varphi_{n I m_{I}}(\xi)
$$


If we expand the total wave function with this basis as

$$
\Psi_{J}(\boldsymbol{R}, \xi)=\sum_{n, L, I} \frac{u_{n L I}^{J}(R)}{R}<\Omega \xi \mid(n L I) J M>
$$

the coupled-channels equations for $u_{n L I}^{J}(R)$ read

$$
\left[-\frac{\hbar^{2}}{2 \mu} \frac{d^{2}}{d R^{2}}+\frac{L(L+1) \hbar^{2}}{2 \mu R^{2}}+U(R)-E+\epsilon_{n I}\right] u_{n L I}^{J}(R)+\sum_{n^{\prime}, L^{\prime}, I^{\prime}} V_{n L I ; n^{\prime} L^{\prime} I^{\prime}}^{J}(R) u_{n^{\prime} L^{\prime} I^{\prime}}^{J}=0
$$

with $V_{n L I ; n^{\prime} L^{\prime} I^{\prime}}^{J}(R)=<J M(n L I)|V(\boldsymbol{R}, \xi)|\left(n^{\prime} L^{\prime} I^{\prime}\right) J M>$. We have suppressed the index $M$ in $V_{n L I ; n^{\prime} L^{\prime} I^{\prime}}^{J}(R)$, since they are independent of that quantum number. These coupledchannels equations are solved with the boundary conditions

$$
u_{n L I}^{J}(R) \rightarrow \frac{1}{T_{n L I}^{J}} H_{L}^{(-)}(R) \delta_{n, n_{i}} \delta_{L, L_{i}} \delta_{I, I_{i}}+\frac{R_{n L I}^{J}}{T_{n L I}^{J}} H_{L}^{(+)}(R) \quad(R \rightarrow \infty)
$$

where $H_{L}^{(+)}(R)$ and $H_{L}^{(-)}(R)$ are the outgoing and the incoming Coulomb waves, respectively. Once the coefficients in the asymptotic region $T_{n L I}^{J}$ are obtained, the penetration probability through the Coulomb potential barrier is given by

$$
P_{L_{i} I_{i}}^{J}(E)=\sum_{n, L, I} \frac{k_{n I}}{k_{n_{i} I_{i}}}\left|T_{n L I}^{J}\right|^{2}
$$

where $k_{n I}=\sqrt{\frac{2 \mu}{\hbar^{2}}\left(E-\epsilon_{n I}\right)}$. The fusion cross section for an unpolarized target is then given by

$$
\sigma_{\text {fus }}(E)=\frac{\pi}{\left(k_{n_{i} I_{i}}\right)^{2}} \sum_{J M L_{i}} \frac{P_{L_{i} I_{i}}^{J}(E)}{2 I_{i}+1}=\frac{\pi}{\left(k_{n_{i} I_{i}}\right)^{2}} \sum_{J L_{i}} \frac{2 J+1}{2 I_{i}+1} P_{L_{i} I_{i}}^{J}(E)
$$

We now introduce the path integral representation of the penetration probability for our coupled-channels problem. Since heavy-ion fusion reactions are processes where two nuclei approach close to each other, we shall treat the radial component of the relative motion as the macroscopic degree of freedom and its angular part and the internal degrees of freedom as environmental degrees of freedom. The barrier transmission probability is 
then given by [17]

$$
\begin{aligned}
P_{L_{i} I_{i}}^{J}(E) & =\lim _{\substack{R_{i} \rightarrow \infty \\
R_{f} \rightarrow-\infty}}\left(\frac{P_{i} P_{f}}{\mu^{2}}\right) \int_{0}^{\infty} d T e^{(i / \hbar) E T} \int_{0}^{\infty} d \widetilde{T} e^{-(i / \hbar) E \widetilde{T}} \\
& \times \int \mathcal{D}[R(t)] \int \mathcal{D}[\widetilde{R}(\tilde{t})] e^{(i / \hbar)\left[S_{t}(R, T)-S_{t}(\widetilde{R}, \widetilde{T})\right]} \rho_{M}(\widetilde{R}(\tilde{t}), \widetilde{T} ; R(t), T),
\end{aligned}
$$

where $P_{i}$ and $P_{f}$ are the classical momenta at the initial and the final positions $R_{i}$ and $R_{f}$, respectively. $S_{t}(R, T)$ is the action for the translational motion along a path $R(t)$, and is given by

$$
S_{t}(R, T)=\int_{0}^{T} d t\left(\frac{1}{2} \mu \dot{R}(t)^{2}-U(R(t))\right)
$$

The effects of the environmental degrees of freedom are included in the two time influence functional $\rho_{M}$, which is defined by

$$
\rho_{M}(\widetilde{R}(\tilde{t}), \widetilde{T} ; R(t), T)=<\left(n_{i} L_{i} I_{i}\right) J M\left|\hat{u}^{\dagger}(\widetilde{R}(\tilde{t}), \widetilde{T}) \hat{u}(R(t), T)\right|\left(n_{i} L_{i} I_{i}\right) J M>
$$

with

$$
i \hbar \frac{\partial}{\partial t} \hat{u}(R, t)=\left[\frac{\boldsymbol{L}^{2} \hbar^{2}}{2 \mu R^{2}}+H_{0}(\xi)+V(R, \Omega, \xi)\right] \hat{u}(R, t) .
$$

$\hat{u}(R, t)$ is the time evolution operator of the environmental degrees of freedom along a given path $R(t)$. The formal solution of eq.(13) can be written as

$$
\hat{u}(R, t)=\hat{T} \exp \left[\int_{0}^{t} d t^{\prime}\left(\frac{\boldsymbol{L}^{2} \hbar^{2}}{2 \mu R\left(t^{\prime}\right)^{2}}+H_{0}(\xi)+V\left(R\left(t^{\prime}\right), \Omega, \xi\right)\right)\right]
$$

where $\hat{T}$ is the time ordering operator. Hereafter the time ordering is supposed to be properly treated in all solutions of $\hat{u}$, and we shall not write it explicitly.

We now assume that the angular part of the translational motion is much slower than the radial motion, and replace the operator $\boldsymbol{L}^{2}$ in eq.(14) by some c-number $\Lambda(\Lambda+1)$ [12]. This is a kind of sudden approximation and is exact if there is no angular momentum transfer from the relative motion between heavy-ions to nuclear intrinsic motion. $\Lambda$ can 
be any c-number, though one often takes $\Lambda$ to be the total angular momentum $J$. If we denote the coordinate representation of $\Omega$ by $\Omega^{\prime}[12$, we get

$$
\begin{aligned}
<\Omega^{\prime}|\hat{u}(R, T)|\left(n_{i} L_{i} I_{i}\right) J M>= & \exp \left[\int_{0}^{T} d t^{\prime}\left(\frac{\Lambda(\Lambda+1) \hbar^{2}}{2 \mu R\left(t^{\prime}\right)^{2}}+H_{0}(\xi)+V\left(R\left(t^{\prime}\right), \Omega^{\prime}, \xi\right)\right)\right] \\
& \times \sum_{m_{L}, m_{I}}<L_{i} m_{L} I_{i} m_{I}\left|J M>Y_{L_{i} m_{L}}\left(\Omega^{\prime}\right)\right| \varphi_{n_{i} I_{i} m_{I}}>\quad(15)
\end{aligned}
$$

We next make a rotational coordinate transformation in the whole space to the coordinate system where the $z$ axis is along the direction of the radial vector $\boldsymbol{R}^{\prime}=\left(R^{\prime}, \Omega^{\prime}\right)=$ $\left(R^{\prime}, \theta^{\prime}, \phi^{\prime}\right)$ [9]. We call the new coordinate system the rotating frame $(\mathrm{RF})$ in order to distinguish it from the space fixed frame(SF). The operator for this coordinate transformation is given by

$$
\mathcal{R}\left(\phi^{\prime}, \theta^{\prime}, 0\right)=e^{i \boldsymbol{J} \cdot \boldsymbol{\chi}\left(\Omega^{\prime}\right) / \hbar}
$$

In eq.(16) $\chi$ is the rotation vector which specifies the direction and the magnitude of the rotation. Note that the third Euler angle in this rotating frame is zero. Since the time evolution operator $\hat{u}(R, t)$ (see eq.(14)) does not change by rotation in the no-Coriolis approximation, we obtain

$$
\begin{aligned}
<\Omega^{\prime}|\hat{u}(R, T)|\left(n_{i} L_{i} I_{i}\right) J M>= & <\Omega^{\prime} \mid \mathcal{R}^{-1}\left(\phi^{\prime}, \theta^{\prime}, 0\right) \mathcal{R}\left(\phi^{\prime}, \theta^{\prime}, 0\right) \hat{u}(R, T) \\
& \times \mathcal{R}^{-1}\left(\phi^{\prime}, \theta^{\prime}, 0\right) \mathcal{R}\left(\phi^{\prime}, \theta^{\prime}, 0\right) \mid\left(n_{i} L_{i} I_{i}\right) J M> \\
= & \sum_{K} \bar{u}(R(t), T)<\Omega^{\prime}=0 \mid\left(n_{i} L_{i} I_{i}\right) J K>D_{K M}^{J}\left(\phi^{\prime}, \theta^{\prime}, 0\right) \\
= & \sum_{K} \bar{u}(R(t), T)<L_{i} 0 I_{i} K\left|J K>\sqrt{\frac{2 L_{i}+1}{4 \pi}} D_{K M}^{J}\left(\phi^{\prime}, \theta^{\prime}, 0\right)\right| \varphi_{n_{i} I_{i} K}>
\end{aligned}
$$

where $D_{K M}^{J}$ is Wigner's $D$ function and the time evolution operator in the rotating frame $\bar{u}(R, T)$ is defined as

$$
\bar{u}(R, T)=\exp \left[\int_{0}^{T} d t^{\prime}\left(\frac{\Lambda(\Lambda+1) \hbar^{2}}{2 \mu R\left(t^{\prime}\right)^{2}}+H_{0}(\xi)+V\left(R\left(t^{\prime}\right), \Omega=0, \xi\right)\right)\right]
$$




$$
=\exp \left[\int_{0}^{T} d t^{\prime}\left(\frac{\Lambda(\Lambda+1) \hbar^{2}}{2 \mu R\left(t^{\prime}\right)^{2}}+H_{0}(\xi)+\sum_{\lambda>0} \sqrt{\frac{2 \lambda+1}{4 \pi}} f_{\lambda}\left(R\left(t^{\prime}\right)\right) T_{\lambda 0}(\xi)\right)\right]
$$

In order to obtain eq.(19) we used

$$
\mathcal{R}\left(\phi^{\prime}, \theta^{\prime}, 0\right)\left|\Omega^{\prime}>=\right| \Omega^{\prime}=0>
$$

and

$$
<\Omega^{\prime}=0 \mid Y_{L_{i} m_{L}}>=\sqrt{\frac{2 L_{i}+1}{4 \pi}} \delta_{m_{L}, 0}
$$

The two time influence functional then becomes

$$
\begin{aligned}
\rho_{M}(\widetilde{R}(\tilde{t}), \widetilde{T} ; R(t), T)= & \int \sin \theta^{\prime} d \theta^{\prime} d \phi^{\prime}<\left(n_{i} L_{i} I_{i}\right) J M\left|\hat{u}^{\dagger}(\widetilde{R}(\tilde{t}), \widetilde{T})\right| \Omega^{\prime}> \\
& \times<\Omega^{\prime}|\hat{u}(R(t), T)|\left(n_{i} L_{i} I_{i}\right) J M> \\
= & \sum_{K} \frac{2 L_{i}+1}{2 J+1}\left|<L_{i} 0 I_{i} K\right| J K>\left.\right|^{2} \\
& \times<\varphi_{n_{i} I_{i} K}\left|\bar{u}^{\dagger}(\widetilde{R}(\tilde{t}), \widetilde{T}) \bar{u}(R(t), T)\right| \varphi_{n_{i} I_{i} K}>
\end{aligned}
$$

In obtaining eq.(25) from eq.(24) we used the orthogonality of the $D$ function

$$
\int \sin \theta d \theta d \phi D_{K^{\prime} M}^{J^{*}}(\phi, \theta, 0) D_{K M}^{J}(\phi, \theta, 0)=\frac{4 \pi}{2 J+1} \delta_{K, K^{\prime}}
$$

The time evolution operator in the rotating frame $\bar{u}(R(t), t)$ obeys

$$
i \hbar \frac{\partial}{\partial t} \bar{u}(R, t)=\left[\frac{\Lambda(\Lambda+1) \hbar^{2}}{2 \mu R^{2}}+H_{0}(\xi)+\sum_{\lambda>0} \sqrt{\frac{2 \lambda+1}{4 \pi}} f_{\lambda}(R) T_{\lambda 0}(\xi)\right] \bar{u}(R, t) .
$$

Eq.(27) shows that the $z$ component of the internal spin $m_{I}$ is conserved in the no-Coriolis approximation. Since the wave functions with different values of $m_{I}$ never couple to each other during the reaction process, the dimension of the coupled-channels equations is drastically reduced. The effective Hamiltonian in eq.(27) has the same form as that in the system where the internal spin is zero. The effects of the finite intrinsic spin enters only through a scaling factor $\sqrt{\frac{2 \lambda+1}{4 \pi}}$ of the coupling strength. 
From eqs.(19), (10), and (25), the fusion cross section in the no-Coriolis approximation finally becomes

$$
\begin{aligned}
\sigma_{f u s}(E)= & \frac{\pi}{\left(k_{n_{i} I_{i}}\right)^{2}} \sum_{J L_{i}} \sum_{K} \frac{2 L_{i}+1}{2 I_{i}+1}\left|<L_{i} 0 I_{i} K\right| J K>\left.\right|^{2} \\
& \times \lim _{\substack{R_{i} \rightarrow \infty \\
R_{f} \rightarrow-\infty}}\left(\frac{P_{i} P_{f}}{\mu^{2}}\right) \int_{0}^{\infty} d T e^{(i / \hbar) E T} \int_{0}^{\infty} d \widetilde{T} e^{-(i / \hbar) E \widetilde{T}} \\
& \times \int \mathcal{D}[R(t)] \int \mathcal{D}[\widetilde{R}(\tilde{t})] e^{(i / \hbar)\left[S_{t}(R, T)-S_{t}(\widetilde{R}, \widetilde{T})\right]}<\varphi_{n_{i} I_{i} K}\left|\bar{u}^{\dagger}(\widetilde{R}(\tilde{t}), \widetilde{T}) \bar{u}(R(t), T)\right| \varphi_{n_{i} I_{i} K}>
\end{aligned}
$$

If the initial value of the internal spin is zero, the initial angular momentum for the relative motion $L_{i}$ equals $J$, and the summation in eq.(28) becomes simple. The fusion cross section in that case can be calculated by treating as though the relative motion couples to a spinless mode of excitation except for the scaling factor $\sqrt{\frac{2 \lambda+1}{4 \pi}}$ mentioned above. If the initial spin of the internal motion is finite, the influence functional is obtained by first calculating it for a fixed $K$-quantum number, and then by taking sum with the weight following Clebsch-Gordan coefficients.

Before closing the paper, we wish to comment on the applicability of the no-Coriolis approximation. It is known that the no-Coriolis approximation cannot be applied when a long range force, such as the Coulomb interaction, is involved[16,19-21]. Heavy-ion fusion reactions are governed by the behavior of the wave functions in the small region near the Coulomb barrier. The no-Coriolis approximation is, therefore, considered to be a good approximation. On the other hand, if one is interested in the angular distributions of elastic and inelastic scattering, the no-Coriolis approximation fails to give the correct scattering phase shifts. In order to cure the problems in such cases, a prescription of renormalizing the coupling strength has been proposed by several authors [19, 20, 21]. The way of renormalization is, however, not unique and this problem is still unsettled.

In summary, we used the path integral method to reformulate the coupled-channels 
problems in the no-Coriolis approximation. We first ignored the change of the centrifugal potential due to an intrinsic excitation. We then introduced a rotational coordinate transformation into the coordinate system where the $z$ axis is along the direction of the radial vector of the relative motion. We have thus shown that the fusion cross section can be calculated by treating nuclear intrinsic motions as though they do not carry a finite angular momentum. The finite multipolarity of nuclear intrinsic excitation appears as a scaling factor in the coupling strength. Though these results have already been obtained by different methods, the advantages of our path integral formulation are that we can easily apply the same technique to cases where the initial internal spin is not zero, and also where the internal angular momentum is not the spin but the orbital angular momentum. The effect of transfer reactions on heavy ion fusion reactions is one such problem [22].

The work of K.H. was supported by Research Fellowships of the Japan Society for the Promotion of Science for Young Scientists. That of J.R.B. was supported by a Japan Society for Promotion of Science Postdoctoral Fellowship for Foreign Researchers in Japan. A.B.B. acknowledges a fellowship from the Japanese Society for Promotion of Science. This work was supported in part by the Grant-in-Aid for General Scientific Research, Contract No.06640368, in part the Grant-in-Aid for Scientific Research on Priority Areas,Contract No.05243101,from the Japanese Ministry of Education, Science and Culture, in part by the U.S. National Science Foundation Grant No. 9314131 and 9303041 and in part by the U.S. Department of Energy Grant DE-FG05-94ER40827. 


\section{References}

[1] M. Beckerman, Rep.Prog.Phys.51, 1047(1988); Phys. Rep. 129, 145(1985); A.B. Balantekin and N. Takigawa, Rev. Mod. Phys., in preparation.

[2] A.O. Caldeira and A.J. Leggett, Phys.Rev.Lett. 46, 211(1981).

[3] P. Hänggi, P. Talkner,and M. Borkovec, Rev.Mod.Phys. 62, 251(1990), and references therein.

[4] Proceedings of the Fourth International Symposium on Foundations of Quantum Mechanics, edited by M. Tsukada et al., Japanese Journal of Applied Physics Series Vol. 9 ( Publication Office of Japanese Journal of Applied Physics, Tokyo, 1993).

[5] N. Takigawa and K. Ikeda, in Proceedings of the Symposium on The Many Facets of Heavy Ion Fusion Reactions, Argonne National Laboratory Report No. ANL-PHY87-1, 1986, p.613.

[6] M.A. Nagarajan, A.B. Balantekin, and N. Takigawa, Phys.Rev.C34, 894(1986).

[7] O. Tanimura, Phys. Rev. C35, 1600(1987).

[8] H. Esbensen, S. Landowne, and C. Price, Phys. Rev. C36, 1216(1987); Phys. Rev. C36, 2359(1987).

[9] N. Takigawa, Y. Alhassid,and A.B. Balantekin, Phys.Rev.C45, 1850(1992).

[10] Y. Alhassid and N. Takigawa, (unpublished).

[11] J. Gomez-Camacho and R.C. Johnson, J. Phys. G12, L235(1986); J. Phys. G14, 609(1988).

[12] K. Hagino, N. Takigawa, J.R. Bennett and D.M. Brink, Phys. Rev. C in press.

[13] K. Mclenithan and D. Secrest, J. Chem. Phys. 80, 2480(1984).

[14] P. McGuire and D.J. Kouri, J. Chem. Phys. 60, 2488(1974).

[15] K. Moribayashi, S. Takada, and H. Nakamura, J. Chem. Phys. 100, 4284(1994). 
[16] Y. Alhassid, V. Liu, and B. Shao, Phys. Rev. A48, 2832(1993); Phys. Rev. A46, $3865(1992)$.

[17] A.B. Balantekin and N. Takigawa,Ann.Phys.(N.Y.) 160,441(1985).

[18] A.B. Balantekin, J.R. Bennett and N. Takigawa, Phys. Rev. C44, 145 (1991).

[19] N. Takigawa, F. Michel, A.B. Balantekin, and G. Reidemeister, Phys. Rev. C44, 477(1991).

[20] Y. Alhassid and H. Attias, Nucl. Phys. A577, 709(1994).

[21] J. Gomez-Camacho, M.V. Andrès, and M.A. Nagarajan, Nucl. Phys. A580, 156(1994).

[22] H. Esbensen and S. Landowne, Nucl. Phys. A492, 473(1989). 